\title{
Bases sociales y psicopolíticas del prejuicio hacia personas trans en habitantes de Córdoba, Argentina
}

\author{
Social and psycho-political basis of prejudice towards trans people \\ among people from Córdoba/Argentina
}

\author{
José Ignacio Páez ${ }^{1}$, Hugo H. Rabbia², Guillermo Erwin Hevia ${ }^{3}$, Florencia Pesci ${ }^{4}$
}

\begin{abstract}
RESUMEN
El prejuicio hacia personas trans se encuentra relativamente poco explorado en comparación a otros prejuicios sexuales y de género. El presente estudio analiza factores explicativos de las actitudes negativas hacia personas trans en ciudadanos/as de Córdoba, Argentina, a partir de la Escala de Actitudes Negativas hacia personas Trans (EANT). Se aplicó un cuestionario a una muestra no aleatoria estratificada $(\mathrm{N}=410)$ de población general. A partir de correlaciones y análisis de path, se replican resultados obtenidos por estudios en otros contextos, destacándose las contribuciones de edad, nivel socioeconómico, intensidad religiosa, variables psicopolíticas (Autoritarismo de Ala Derecha [RWA], Orientación hacia la Dominancia Social [SDO] - subfactor dominancia grupal), y psicosociales (sexismo y prejuicio sexual hacia gays y lesbianas, indagado con la Escala de Actitudes hacia Gays y Lesbianas [ATGL]). El modelo de path propuesto, explicó el $65 \%$ de la varianza total de la EANT, y presentó buenos ajustes globales. El artículo discute también las relaciones entre la EANT y ATGL.
\end{abstract}

Palabras clave: Prejuicio, Actitudes, LGBT, Sexismo, Psicología Política.

\begin{abstract}
The prejudice toward trans people remains relatively unexplored compared to other sexual and gender prejudices. This study analyzes the explanatory factors of prejudice towards trans people in Cordoba, Argentina, through the Negative Attitudes toward Trans Scale (EANT, in Spanish). We applied a questionnaire to a stratified non-random sample $(\mathrm{N}=410)$ of general population, to explore an explanatory model of EANT. From correlations and path analysis, we replicated results from studies in other contexts, highlighting the contributions of age, socioeconomic status, religiousness, psycho-political (Right Wing Authoritarism [RWA], Social Dominance Orientation [SDO] - group dominance) and psycho-social prejudice measures (ambivalent sexism and attitudes toward gays and lesbians, ATGL). The proposed model explained $65 \%$ of the total variance of the EANT and reported good global settings. The paper also discusses the relationship between EANT and ATGL.
\end{abstract}

Keywords: Prejudice, Attitudes, LGBT, Sexism, Political Psychology.
Recibido: $21 / 09 / 2018$ Aceptado: 15/10/2018
Citar: Páez, J. I., Rabbia, H. H., Hevia, G. E., \& Pesci, F. (2018). Bases sociales y psicopolíticas del prejuicio hacia personas trans en habitantes de Córdoba, Argentina. Investigaciones en Psicología, 23(2), pp. 27-36. doi:10.32824/investigpsicol.a23n2a3

1Universidad Nacional de Córdoba. Facultad de Psicología. Córdoba, Argentina. paezjoseunc@gmail.com

${ }^{2}$ CONICET - Universidad Nacional de Córdoba. Facultad de Psicología. Instituto de Investigaciones Psicológicas (IIPsi). Córdoba, Argentina. hrabbia@conicet.gov.ar

${ }^{3}$ Universidad Nacional de Córdoba. Facultad de Psicología. Córdoba, Argentina.

${ }^{4}$ Universidad Nacional de Córdoba. Facultad de Psicología. Córdoba, Argentina. 
Bases sociales y psicopolíticas del prejuicio hacia
personas trans en habitantes de Córdoba, Argentina

En mayo de 2012 el Senado Argentino aprobó la Ley $\mathrm{N}^{\circ}$ 26.743, conocida como Ley de Identidad de Género, con el objeto de garantizar a personas trans ${ }^{1}$ (travestis, transexuales, transgéneros) el reconocimiento pleno de su identidad de género y el goce de su salud integral. La legislación no requiere intervención médica, psiquiátrica y/o judicial a las personas que soliciten la readecuación registral de su nombre y género, a la vez que garantiza acceso gratuito a tratamientos hormonales, intervenciones quirúrgicas totales o parciales para adecuar su cuerpo a su identidad de género autopercibida. Por ello, ha sido caracterizada como una ley "de vanguardia” por organizaciones y movimientos sociales que luchan por los derechos de personas trans en el mundo (Schmall, 2012).

La Ley de Identidad de Género constituye un eslabón relevante en una cadena de conquistas legislativas conseguidas por activistas de la diversidad sexual a lo largo de las últimas dos décadas en el país, como la Ley de Educación Sexual Integral de 2006 y la Ley de Matrimonio Igualitario de 2010. A su vez, se presenta como una oportunidad inicial sin precedentes para posibilitar el acceso y ejercicio de derechos humanos básicos a uno de los sectores de la población más vulnerados. Según numerosos estudios, las personas trans están sujetas con frecuencia a actos de discriminación, violencia y hostigamiento de todo tipo, interpersonal e institucional, médica, psicológica y física, a la vez que sufren serias dificultades de acceso a sistemas de salud, educación y trabajo (INDEC, 2012; Iosa, Rabbia, Sgró Ruata, Morán Faúndes \& Vaggione, 2012).

Si bien algunos estudios han señalado que este ciclo de reconocimiento de derechos sexuales se acompaña por una mayor aceptación social de gays y lesbianas en diversas sociedades latinoamericanas (Cárdenas \& Barrientos, 2008; Jorge, 2012), no existen evidencias sistemáticas sobre las dinámicas de las actitudes sociales hacia personas trans. Por el contrario, acontecimientos recientes, como las polémicas generadas en torno a proyectos legislativos de subsidios reparativos para personas trans y de cuotas de inserción laboral para varones trans (Bazán, 2014), así como los debates en torno a la efectiva inclusión de contenidos de género y diversidad sexual en la educación sexual (Theumer, 2018), sugieren que el prejuicio sexual, la transfobia y la estigmatización social conti-

${ }^{1}$ En el presente trabajo se incluye bajo la órbita del término "trans" a personas transexuales, travestis y transgéneros. Esta definición, aunque insuficiente, permite cierta operativización analítica. El conocimiento y creencias asociadas a la noción "trans" que evocan espontáneamente las personas entrevistadas fue recogido por la presente investigación. Si bien no forman parte de esta comunicación, cabe señalar que las creencias relevadas apuntan a una creciente homogeneización en el sentido común dominante de los conceptos transexuales, travestis y transgénero, a la vez que un reconocimiento del concepto "trans" como marcador identitario central de las luchas políticas en las que se ven insertas sus demandas. núan vigentes

De allí que el objetivo del presente artículo es avanzar en el análisis de los factores explicativos de las actitudes negativas hacia personas trans, a la vez que identificar particularidades respecto de éstas con el prejuicio hacia personas gays y lesbianas, a partir de datos obtenidos por cuestionarios aplicados a residentes en la ciudad de Córdoba, Argentina.

\section{El estudio del prejuicio hacia personas trans}

La investigación sobre actitudes negativas y prejuicio hacia personas trans es relativamente reciente. En 2002, Hill señaló que el estudio de la violencia anti-transgénero era "débil" debido a la carencia de un marco conceptual desde el cual entender el rechazo dirigido hacia esta comunidad. Desde entonces, se han registrado diversos estudios sobre transfobia, actitudes negativas y prejuicio hacia personas trans (Hill \& Willoughby, 2005; Nagoshi, et al., 2008; Norton \& Herek, 2012), aunque aún resulta un campo poco explorado. En comparación, existe una tradición más sólida y continuada de estudios de las actitudes hacia personas gays y lesbianas, la homofobia y la homonegatividad (Cárdenas \& Barrientos, 2008; Kite \& Whitley, 1996; Herek, 1988, 2000; Moral de la Rubia, 2010).

En términos operativos, existen instrumentos para abordar el prejuicio hacia personas trans. Hilly Willoughby (2005) han desarrollado la Escala de Ideología de Género y Transfobia (GTS, del inglés Genderism and Transphobia Scale), la cual reporta el rechazo emocional (disgust), violencia, hostigamiento y discriminación hacia personas trans. Los autores identifican una triple dimensionalización del prejuicio antitrans en las dimensiones transfobia, hostigamiento de género (gender-bashing) e ideología de género (genderism), aunque la escala presenta algunos problemas psicométricos y su estructura factorial subyacente admite dos factores: una dimensión afectivo-cognitiva, ideología de género/transfobia, y una comportamental, hostigamiento de género.

Nagoshi, et al. (2008) han realizado diversas críticas psicométricas y teóricas a la GTS y afirman que "una medida más corta y ajustada podría capturar toda la varianza relevante de la escala original” (p. 522). Por ello, proponen una escala de transfobia de nueve ítems que privilegia los componentes afectivos sobre los cognitivos, la cual ha sido validada en población estudiantil (Nagoshi et al., 2008).

Otros estudios han utilizado un único reactivo, como el termómetro de sentimientos (Norton \& Herek, 2012), o la auto-ubicación de las personas entrevistadas respecto a cuán positiva o negativa consideran la presencia de "grupos transgéneros” en la sociedad (Rottenbacher, 2012).

A partir de considerar fraseos de reactivos típicos de escalas de prejuicio sexual previas, Páez, Hevia, Pesci y Rabbia (2015) han construido y validado con población estudiantil y población general la Escala de Actitudes Negativas hacia personas Trans (EANT, por su sigla en castellano), adecuada al contexto local. La escala, que 
presenta excelentes indicadores psicométricos, refleja de manera unidimensional las posiciones de rechazo manifiesto a personas trans a partir de 9 reactivos que se responden en función del nivel de acuerdo con cada uno de ellos. La EANT ha sido replicada en algunos estudios en la región, pero sólo con población estudiantil (Rodríguez Otero \& Treviño Martínez, 2016).

\section{1.a Relaciones entre prejuicio sexual y actitudes hacia personas trans}

Si bien diversos reactivos de las escalas de homonegatividad, actitudes hacia gays, lesbianas y bisexuales, y actitudes hacia personas trans tienden a ser similares, no existe acuerdo sobre la conveniencia de la diferenciación entre el prejuicio sexual (o por orientación sexual, es decir, hacia gays, lesbianas y/o bisexuales) y el prejuicio hacia personas trans.

En su meta-análisis sobre estudios de prejuicio sexual, Kite y Whitley (1996) abordaron la idea de un sistema de creencias de género como constructo integrador de los diversos estereotipos sobre varones y mujeres y actitudes respecto a los roles de género socialmente esperados, así como las percepciones y actitudes hacia aquellas personas que supuestamente disienten o cuestionan las normativas de género en cada sociedad. Whitley \& Ægisdottir (2000) afirmaron que las creencias de roles de género actuaban como mitos legitimadores del prejuicio sexual.

En esta línea, Costa, Bandeira y Nardi (2015) han propuesto la Escala de Prejuicio hacia la Diversidad Sexual y de Género (PASGD, del inglés Prejudice Against Sexual and Gender Diversity), la cual integra la Escala de Actitudes hacia Gays y Lesbianas (ATGL, del inglés Attitudes Toward Gay and Lesbian) de Herek y McLemore (2011), y la GTS de Hill y Willoughy (2005), con algunos ítems adaptados al contexto brasileño. A partir de una versión revisada de la escala (Costa, Machado, Bandeira \& Nardi, 2016), y considerando los resultados de los análisis factoriales y las fuertes correlaciones entre prejuicio de género y prejuicio sexual $(r .98, p<.001)$ que caracteriza a la muestra brasileña (a diferencia de las estadounidenses), los autores sugieren la presencia de un único factor general donde confluyen ambos prejuicios.

En contraposición, otros estudios han señalado la necesidad de diferenciar conceptual y empíricamente el prejucio hacia personas trans respecto del prejuicio sexual. Para Herek, Chopp y Stroll (2007) esta diferencia se debe a que el prejuicio hacia personas trans implica a las concepciones dominantes de género y no una matriz de diferenciación sexual. Worthen (2013), en igual sentido, ha enfatizado la necesidad de un estudio diferenciado del prejuicio hacia gays, lesbianas, bisexuales, y trans (sean femeninos a masculinos, o masculinos a femeninos), puesto que las intersecciones de género y orientación sexual afectarían las actitudes hacia esta población, de manera particular para cada caso. Diversos estudios sobre brechas de género en la evaluación del prejuicio sexual generizado -diferenciado entre varones gays y mujeres lesbianas- (Herek, 2002), así como sobre bifobia entre activistas gays y lesbianas (Weltzer-Lang, 2008, en
Worther, 2013) validan esta perspectiva, aunque cada una de estas escalas actitudinales tiendan a presentar fuertes relaciones entre sí.

Al comparar en estudiantes de trabajo social mexicanos los niveles de sexismo (utilizando la escala de sexismo ambivalente de Glick y Fiske [1996]), homofobia (con la Escala Mexicana de Homofobia, EHF-10, de Moral de la Rubia y Valle de la O. [2011]), las actitudes hacia la bisexualidad (según el instrumento propuesto por Olvera [2014]) y la EANT, Rodríguez Otero y Reviño Martínez (2016) advierten factores explicativos y niveles similares de bifobia, transfobia y homofobia, aunque más altos de sexismo. A su vez, la correlación entre la escala de homofobia y la EANT, si bien alta $(r .77, p<.001)$, no alcanzan los niveles identificados en la muestra brasileña por Costa et al., (2016) o en un estudio realizado en Grecia, donde la ATGL se analizó como variable independiente de la GTS $(r .83, p<.001)$ (Grigoropoulos \& Kordoutis, 2015). Además, la mayoría de los trabajos sobre actitudes hacia personas trans reportan variables sociodemográficas, psicosociales y sociopolíticas explicativas similares a las del prejuicio hacia gays y lesbianas (Herek, 2000).

\section{1.b. Prejuicio hacia personas trans y variables socio- demográficas}

Cualquiera sea el instrumento utilizado, los estudios identifican que las mujeres heterosexuales presentan menores niveles de actitudes negativas hacia personas trans que los varones (Costa et al., 2016; Costa \& Davies, 2012; Grigoropoulos \& Kordoutis, 2015; Hill \& Willoughby, 2005; Landen \& Innala, 2000; Norton \& Herek 2012). Respecto de la variable edad, las evidencias no son consistentes: Norton y Herek (2012) no encontraron diferencias significativas en los niveles de prejuicio según la edad, mientras que Landen e Innala (2000) reportan que las personas más jóvenes presentan menores niveles de actitudes negativas hacia trans que las de mayor edad. Norton y Herek (2012) advierten también que las personas con menor nivel educativo y de ingresos tienden a presentar mayores niveles de actitudes negativas hacia personas trans, replicándose resultados similares a los relevados en otros tipos de prejuicio.

\section{1.c. Prejuicio hacia personas trans y religiosidad}

Existen evidencias coincidentes de una relación positiva entre religiosidad personal y prejuicio sexual y prejuicio de género. Nagoshi et al. (2008) estudian el fundamentalismo religioso, encontrando fuertes correlaciones positivas con medidas de transfobia. Norton y Herek (2012) indagan la importancia que las personas atribuyen a la religión en sus propias vidas, siendo que quienes le otorgan mayor importancia presentan mayores niveles de prejuicio sexual hacia personas trans. Otros trabajos identificaron una fuerte relación positiva entre religiosidad y transfobia, realizando el análisis a través de medidas de pertenencia religiosa, religiosidad auto-percibida y/o frecuencia de asistencia al culto (Costa et al., 2016; Willoughby et al., 2010). 


\section{1.d Prejuicio hacia personas trans y variables psico- políticas e ideológicas}

Estudios recientes han sugerido que la relación entre la religiosidad personal y el prejuicio sexual o el sexismo es indirecta, siendo ésta mediada por variables psicopolíticas y de personalidad, como la necesidad de cierre cognitivo, el Autoritarismo de Ala Derecha (RWA, del inglés: Right Wing Authoritarism) o la SDO (Orientación a la Dominancia Social, del inglés: Social Dominance Orientation [Johnson, et al., 2011; Hill, Cohen, Terrell, \& Nagoshi, 2010]). Diversos estudios han identificado relaciones positivas entre RWA, SDO y el prejuicio sexual (Burke et al., 2017; Poteat \& Mereish, 2012). En Argentina, se ha replicado estos resultados aplicando una versión localmente validada de la ATG (Etchezahar, Ungaretti, Prado \& Brussino, 2016).

El RWA se basa en la necesidad de mantener las tradiciones sociales y resulta de la covariación de tres componentes actitudinales: la sumisión autoritaria (entendida como un alto grado de aceptación y legitimización de las acciones de las autoridades, incluso cuando las mismas son injustas o corruptas), la agresión autoritaria (predisposición a perjudicar a personas de determinados grupos, con la convicción de que el poder establecido lo aprueba) y el convencionalismo (alto nivel de aceptación de las normas vigentes [Altemeyer, 2004]). Algunos estudios relacionan el RWA con el prejuicio hacia personas trans, encontrando que las personas más autoritarias presentan mayores niveles de rechazo hacia personas trans (Nagoshi et al., 2008; Rottenbacher, 2010; Norton \& Herek, 2012).

La SDO es definida por Pratto, Sidanius, Stallworth y Malle (1994) como una variable ideológica de la personalidad que refiere el grado en que una persona tiende a comprender el mundo y la existencia en términos de una permanente competición entre grupos sociales, deseando mantener la estratificación social y promoviendo el dominio de grupos superiores sobre aquellos que consideran inferiores. En su estudio con universitarios peruanos, Rottenbacher (2010) concluyó que las personas que puntúan alto en SDO presentan mayores actitudes negativas hacia personas trans.

A través de la SDO se han evidenciado posiciones conservadoras y jerárquicas respecto al orden social, incluyendo aquellas que refieren a las jerarquías de género: se ha afirmado que los varones puntúan significativamente más alto en el subfactor Orientación a la Dominancia Grupal, aunque no se registran diferencias de género en la Orientación a la Igualdad ni en RWA (Petit \& Costa, 2011).

El sexismo también puede brindar evidencias de posiciones conservadoras sobre el orden de género y sexual. Glick y Fiske (1996) han propuesto la coexistencia simultánea de dos tipos de sexismo: uno hostil y uno benevolente, fundado en el supuesto de que en las sociedades contemporáneas el sexismo ha mutado a manifestaciones sutiles pero que mantienen su motivación principal: la defensa de los roles de género tradicionales en favor del dominio masculino. Así, el sexismo hostil correspondería a las formas tradicionales de prejuicio ya que se compone de actitudes prejuiciosas y conductas discriminatorias basadas en la supuesta inferioridad de la mujer (Cárdenas, Lay, González, Calderón \& Alegría, 2010), mientras que el sexismo benevolente se caracteriza por actitudes aparentemente no prejuiciosas hacia las mujeres que son expresadas en un tono afectivamente positivo, aunque paternalistas e igualmente estereotipadas. Estas actitudes tienden a relacionar a las mujeres con roles socialmente restringidos, lo cual las presenta como personas frágiles, necesitadas de cuidado y protección al mismo tiempo que son concebidas como complemento de los varones.

Algunos estudios han encontrado que la homofobia se relaciona de manera positiva y significativa con las dos sub-escalas de sexismo, siendo la correlación de mayor peso con el sexismo hostil (Rottenbacher, Espinosa \& Magallanes 2011). En su investigación con estudiantes universitarios mexicanos, Rodríguez Otero y Reviño Martínez (2016) advirtieron correlaciones positivas moderadas entre sexismo y la EANT. Por otra parte, RWA y SDO han sido consideradas variables explicativas por excelencia del sexismo, el prejuicio sexual, y otros tipos de prejuicio (Etchezahar, 2012), por lo cual es posible pensar que estos constructos se presenten como mediadores entre las primeras y las actitudes negativas hacia personas trans.

Siendo el sexismo y el prejuicio hacia gays y lesbianas expresiones de posiciones tradicionales del orden de género y sexual, las cuales se verían desafiadas por la transgeneridad, la transexualidad y el no conformismo de género, se considera pertinente avanzar en el análisis de los efectos entre el sexismo hostil y benevolente y ATGL, en las actitudes hacia las personas trans en población general.

\section{Metodología}

\section{Diseño y Participantes}

Se realizó un estudio empírico cuantitativo, ex post facto, por cuestionario. Los/as participantes del estudio fueron seleccionados/as a través de un muestreo no aleatorio estratificado por cuotas (Lohr, 2000) de edad, nivel socioeconómico y sexo asignado al nacer entre residentes en la Ciudad de Córdoba de 18 a 65 años. Si bien este muestreo no garantiza la aleatoriedad de la muestra, se logró una distribución de los casos por cuotas de edad, sexo y nivel socio-económico semejante a las proporciones estimadas por el Instituto Nacional de Estadísticas y Censos (INDEC, 2011) para la ciudad de Córdoba. La muestra quedó confeccionada por 410 personas (198 varones y 212 mujeres) entre 18 y 65 años, siendo la media de edad de 37.52 años (DS $=13.43$ ). Se recogió el consentimiento de los/as participantes, garantizando condiciones de confidencialidad, anonimato y utilización de la información con fines de investigación.

\section{Instrumentos}

Las actitudes hacia personas trans fueron abordadas a través de la Escala de Actitudes Negativas hacia personas 
Trans (EANT) (Paez, et al., 2015). Consta de nueve ítems que indagan prejuicio manifiesto a partir de actitudes negativas fuertemente estereotipadas hacia personas trans, y se responden en función de una escala tipo Likert de cinco puntos. A partir de los puntajes agregados de la EANT (que van de 9 a 45 ) se da cuenta del nivel de actitudes desfavorables hacia personas trans $(\alpha=.88)$.

Sexismo: se tomó una versión adaptada de 22 ítems al contexto local por Etchezahar (2012) del instrumento desarrollado por Glick y Fiske (1996) de sexismo ambivalente. Mayores puntajes en la escala indican mayor nivel de sexismo. La escala posee una estructura bidimensional: sexismo hostil $(\alpha=.84)$ y sexismo benevolente $(\alpha=.89)$.

Prejuicio sexual hacia gays y lesbianas: se aplicaron las formas abreviadas de 3 ítems de las escalas de Actitudes hacia Hombres Gays y Actitudes hacia Lesbianas, propuesta para estudios poblacionales por Herek (1994, 1997). Puntuaciones altas indican altos niveles de prejuicio sexual. Algunos estudios reportan ambas escalas de forma agregada (ATGL), lo cual es conveniente cuando se utiliza como variable independiente (Pearl \& Galuppo, 2007) y en muestras poblacionales. Las escalas sumadas presentaron excelente consistencia interna $(\alpha=.90)$.

Intensidad del sentimiento religioso: según la escala propuesta por Grasmick, Cochran, Bursik Jr. y Kimpel (1993), y validada para el contexto local por Lista (1995). Incluye cinco ítems que van desde la importancia asignada a la religión en la propia vida a la auto-percepción de religiosidad. Altos puntajes indican alta intensidad religiosa. La escala presenta una estructura factorial unidimensional y una buena consistencia interna $(\alpha=.86)$.

Autoritarismo de Ala Derecha (RWA): se utilizó una adaptación al contexto local de la versión española de la escala de la RWA (Seoane \& Garzón, 1992) desarrollada por Imhoff y Brussino (2013). La escala, unidimensional, cuenta con 10 reactivos, pero la adaptación realizada por Imhoff y Brussino (2013) no contiene la dimensión de convencionalismo del original $(\alpha=.89)$.

Orientación hacia la Dominancia Social (SDO): se utilizó una versión de Orientación hacia la Dominancia Social (Pratto, et al., 1994) adaptada por Jaume, Etchezahar, Cervone y Biglieri (2011) al contexto local. Se compone de 10 ítems (5 de Dominancia Grupal y 5 de Oposición a la Igualdad). En Jaume, et al. (2011) la escala presentó indicadores de consistencia bajos de forma global $(\alpha=.31$ para la escala total). Puntajes altos en esta escala, implican mayor Orientación hacia la Dominancia Social. En los análisis posteriores se utilizó sólo la dimensión de Dominancia Grupal, debido a que presentó mejores indicadores estadísticos que la dimensión de Oposición a la Igualdad y la escala en su forma global $(\alpha=.61$ en SDO-Dominancia Grupal, $\alpha=.55$ en SDO-Orientación a la Igualdad y $\alpha=.30$ SDO global), no obstante, presenta indicadores de baja consistencia interna.

También se indagó el sexo registrado al nacer, el Nivel Socio-Económico (NSE) -a partir de la propuesta de evaluación de la Comisión de Enlace Institucional (AAM, SIEMPRO \& CEIM, 2006), el máximo nivel educativo alcanzado por la persona encuestada, y la edad.

\section{Análisis de datos}

Se llevaron a cabo análisis de tablas de contingencia y correlaciones bivariadas, para explorar las relaciones de las variables independientes con la variable dependiente. Se prestó especial atención a los estadísticos Chi cuadrado y $V$ de Cramer (contingencias) y a $r$ de Pearson (correlaciones) para conocer si entre las variables existe asociación y la intensidad de la misma. Se siguieron los criterios establecidos por Pérez-Tejada (2007) para evaluar las fuerzas de las correlaciones. Se operó un filtro para que solo las personas heterosexuales sean contempladas en los análisis, las cuales componen el $94,4 \%$ de la muestra ${ }^{2}$.

Luego se realizó un análisis de camino (path analysis) con el fin de testear un modelo teórico que permita comprender las actitudes hacia personas trans, integrando las variables en estudio a partir de las propuestas de los antecedentes relevados. Si bien esta técnica no implica un análisis causal dado que no se pueden controlar otras variables del entorno, permite indagar los efectos directos e indirectos de las variables determinantes en la variable endógena pura (EANT) y contribuir a su explicación. Para el ajuste del modelo se tuvieron en cuenta los coeficientes globales de ajuste, los de estimaciones estandarizadas y los valores de parsimonia, recomendada por diversos autores (Kline, 2011; Pérez, Medrano \& Sánchez Rosas, 2013). A su vez, en el modelo teórico testeado se procedió a eliminar los enlaces con estimaciones no significativas ( $p \leq .05) \mathrm{y}$, en caso de ser coincidente con las propuestas teóricas, se adoptaron nuevos enlaces según los índices de modificación propuestos por el programa estadístico.

Dada la discusión sobre la relación entre prejuicio sexual y prejuicio hacia personas trans, se contrastó el modelo teórico inicial con un segundo modelo donde la variable endógena pura se conformó a partir de una variable latente que incluyera tanto el prejuicio sexual (ATGL) como las actitudes negativas hacia personas trans (EANT). Dicha variable se denominó Actitudes negativas hacia trans, gays y lesbianas (ANTGL). Se consideraron los indicadores recomendados por Pérez et al., (2013) para comparar los dos modelos re-especificados.

\section{Resultados}

La media del puntaje agregado de la EANT fue de $28.03(\min =9$ y $\max =45)$, un prejuicio moderado, aunque tendiendo a actitudes negativas hacia personas trans. Entre personas auto-identificadas como heterosexuales, la media fue de 28.17 , siendo más alta en personas que fueron asignadas como varones al nacer (29.25) que en mujeres (25.30), aunque esta diferencia no es significativa estadísticamente. Entre las personas no

${ }^{2}$ Como se señaló previamente, esto no implica que sólo las personas auto-identificadas como heterosexuales expresen prejuicio hacia personas trans, pero el número total de personas que se auto-identificaron como "gay”, "lesbiana”, "bisexual”, no supone un número comparable en términos estadísticos con las que se identificaron como "heterosexuales". 
heterosexuales la media de actitudes negativas hacia personas trans desciende a 13.30.

Como se advierte en la Tabla 1, todas las correlaciones entre la EANT y las variables independientes resultaron significativas estadísticamente y en el sentido esperado. Las correlaciones son positivas, con excepción del nivel socioeconómico y el nivel educativo en los que se registró una correlación inversa (es decir, a menor nivel socioeconómico y menor nivel educativo, mayor prejuicio hacia personas trans). Las correlaciones positivas, aunque moderadas, entre RWA, intensidad religiosa, y sexismo hostil, así como las correlaciones positivas y fuertes entre sexismo benevolente, ATGL y la EANT, evidencian resultados similares a los registrados por los antecedentes. Las personas con mayor intensidad del sentimiento religioso, cualquiera sea su auto-adscripción religiosa, manifiestan un prejuicio mayor hacia personas trans, más sexismo (benevolente y hostil) y más prejuicio sexual (ATGL), en consonancia con las personas que manifiestan un alto nivel de autoritarismo. En este punto, resulta llamativo que el sexismo benevolente o sutil presente correlaciones más fuertes con medidas de prejuicio manifiesto como las que proponen la EANT y la ATGL. Se advierte, no obstante, correlaciones positivas débiles entre SDO, en su subfactor de Orientación de Dominancia Grupal, y la EANT, así como las otras mediciones de prejuicio indagadas, lo cual sugeriría que la adopción de una ideología personal de jerarquías intergrupales no presentaría el mismo rol explicativo del prejuicio que el sugerido por otras variables psicopolíticas aquí relevadas.

Tabla 1. Correlaciones entre EANT y variables independientes

\begin{tabular}{|c|c|c|c|c|c|c|c|c|c|c|}
\hline & EANT & Edad & NSE & $\begin{array}{c}\text { Nivel } \\
\text { Educat. }\end{array}$ & Int. Relig. & RWA & $\begin{array}{l}\text { SDO } \\
\text { DG }\end{array}$ & $\begin{array}{l}\text { Sex. } \\
\text { Hostil }\end{array}$ & $\begin{array}{c}\text { Sex } \\
\text { Benev }\end{array}$ & ATGL \\
\hline EANT & 1 & & & & & & & & & \\
\hline Edad & $.284^{* *}$ & 1 & & & & & & & & \\
\hline NSE & $-.387^{* *}$ & .041 & 1 & & & & & & & \\
\hline Nivel Educat. & $-.134^{* *}$ & $-.104^{* *}$ & $.174^{* *}$ & 1 & & & & & & \\
\hline Int. Relig. & $.571^{* *}$ & $.312^{* *}$ & $-.350^{* *}$ & $-.173^{* *}$ & 1 & & & & & \\
\hline RWA & $.538^{* *}$ & $.140^{* *}$ & $-487^{* *}$ & $-.165^{* *}$ & $.497^{* *}$ & 1 & & & & \\
\hline SDO DG & $.207^{* *}$ & -.018 & -.040 & 0.29 & $.169 * *$ & $.301 * *$ & 1 & & & \\
\hline Sex. Hostil & $.531^{* *}$ & .028 & $-.409 * *$ & $-.123^{* *}$ & $.346^{* *}$ & $.662 * *$ & $.312 * *$ & 1 & & \\
\hline Sex. Benev. & $.676^{* *}$ & $.184^{* *}$ & $-.471^{* *}$ & $-.162^{* *}$ & $.568^{* *}$ & $.708^{* *}$ & $.204^{* *}$ & $.690^{* *}$ & 1 & \\
\hline ATGL & $.771 * *$ & $.296^{* *}$ & $-.374^{* *}$ & $-.110 * *$ & $.562 * *$ & $.513 * *$ & $.211^{* *}$ & $.492 * *$ & $.619^{* *}$ & 1 \\
\hline
\end{tabular}

Tabla de elaboración propia. $\mathrm{N}=387$ Heterosexuales.

${ }^{* *} p \leq .001, * p \leq .05$.

Referencias: EANT = Escala de Actitudes Negativas Hacia Personas Trans (Páez, et. al. 2015); NSE = Nivel Socioeconómico (AAM, SIEMPRO \& CEIM, 2006); Nivel Educat. = Nivel Educativo (AAM, SIEMPRO \& CEIM, 2006); Int. Relig. = Intensidad Religiosa (Lista, 1995); RWA = Autoritarismo de Ala de Derecha (Imhoff \& Brussino, 2013); SDO DG = Dominancia Grupal (dimensión de la Orientación hacia la Dominancia Social) [Jaume, et.al. 2011]; Sex. Hostil = Sexismo Hostil (Etchezahar, 2012); Sex. Benev. = Sexismo Benevolente (Etchezahar, 2012); ATGL $=$ Actitudes hacia Gays y Lesbianas (Herek, 1994, 1997).

A partir del análisis de path o caminos, es posible advertir las contribuciones directas e indirectas de las variables independientes relevadas en las actitudes negativas hacia personas trans. El modelo de path re-especificado (figura 1) explicó el $65 \%$ de la varianza de la variable endógena pura y presentó índices de ajuste adecuados $\left(X^{2}=30.366 p \leq .005, \mathrm{gl}=14 ; X^{2} / \mathrm{gl}=2.169\right.$; $\mathrm{AGFI}=.949 ; \mathrm{CFI}=.991$ y $\mathrm{RMSEA}=.053)$.

La variable que más aportó a la EANT es la dimensión benevolente del sexismo con un $67 \%$ de varianza total aportada, tanto de manera directa como indirecta, en este caso a través de ATGL. Esta última resultó ser la segunda variable con más efecto en la endógena pura con un $52 \%$ de varianza aportada de manera directa. Además, se destaca la dimensión hostil del sexismo ambivalente con un $51 \%$ de varianza aportada mediatizada por la ATGL y el sexismo benevolente. De las variables de conservadurismo político, el RWA presentó un efecto relevante e indirecto en la escala de actitudes hacia personas trans, ya que su contribución es de $44 \%$, mediado por el sexismo hostil y benevolente. Finalmente, la orientacion de dominancia social, en su dimensión de dominancia grupal (SDO_DG), con una contribución del 5\% de varianza explicada a través de sexismo hostil y RWA, como las variables exógenas intensidad religiosa, edad y nivel socioeconómico no aportaron fuertemente en la explicación de las actitudes hacia personas trans, posiblemente debido a que su vínculo con las mismas se encuentran mediatizados a través de otras variables.

El modelo re-especificado final se presenta a continuación, con sus efectos directos estandarizados $(\beta)$ y el coeficiente de determinación $\left(R^{2}\right)$. 
Figura 1. Modelo re-especificado para EANTP

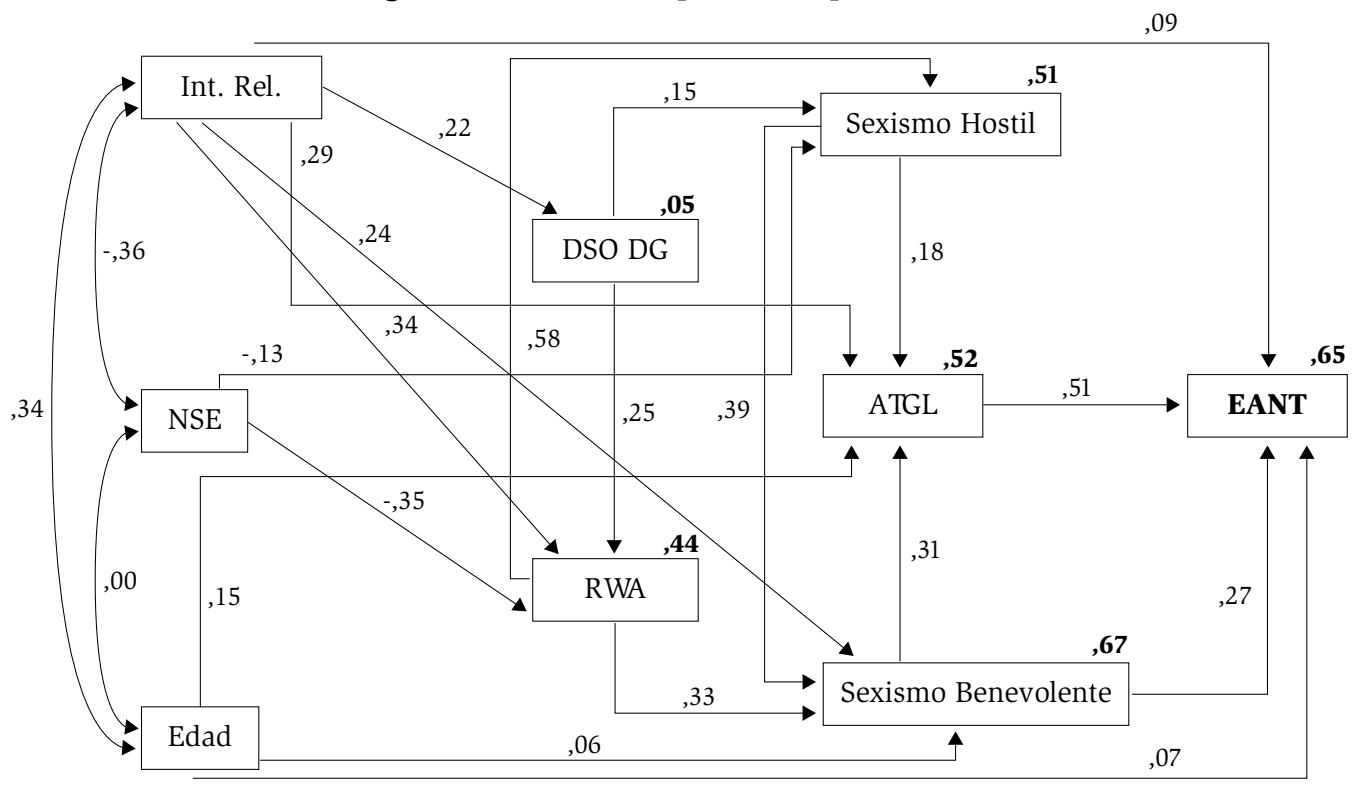

Nota: Se reportan sólo enlaces significativos, los coeficientes estandarizados de cada enlace como así también las proporciones de varianza explicada por cada variable. Referencias: Int. Rel. = Intensidad Religiosa; NSE = Nivel socioeconómico; SDO DG = Dominancia Grupal (dimensión de la Orientación hacia la Dominancia Social); RWA = Autoritarismo de Ala de Derecha; $\mathbf{A T G L}=$ Actitudes hacia gays y lesbianas. Fuente: elaboración propia.

Si bien se registraron resultados que podrían indicar problemas de multicolinealidad con la variable ATGL, cuando ésta es controlada estadísticamente en el path (Ver Tabla 2), a partir de una nueva re-especificación del modelo que no incluya esta variable, el sexismo ambivalente y RWA continúan siendo buenos factores explicativos del prejuicio hacia personas trans, y se mantienen buenos indicadores globales del modelo. Al contrastar el presente modelo con un modelo re-especificado que toma la EANT y la ATGL como componentes de una variable latente de actitudes negativas hacia trans, lesbianas y gays (ANTGL), es posible advertir que, si bien el menor valor en el Criterio de Información de Akaike (AIC) de este último modelo indicaría un relativo mejor ajuste respecto del modelo teórico inicial y del modelo sin ATGL, un RMSEA $p \leq . .08$ no es recomendable porque podría indicar un error de aproximación, lo cual nos hace preferir el modelo teórico identificado inicialmente. De forma global, el modelo ANTGL explicaría un $66 \%$ de la varianza total, siendo que los efectos directos e indirectos y contribuciones de cada variable indagada permanecen de manera similar al modelo teórico inicial.

En la tabla 3 se observa que la variable con mayor efecto directo y total sobre la variable endógena pura $($ EANT) fue la ATGL $(\beta D T=.515)$. En segundo término, se destaca el efecto total del sexismo benevolente $(\beta T=.433)$ y de la religiosidad $(\beta T=.462)$, que si bien se relacionan positivamente con las actitudes hacia personas trans, en el primer caso presenta un efecto directo $(\beta D=.272)$ más fuerte que en el segundo debido a la proximidad teórica de la variable con la endógena pura, en el cual el mayor aporte se produce a través de efectos indirectos $(\beta I=.377)$. Se advierte que el sexismo hostil y las variables ideológicas (SDO-Dominancia Grupal y RWA) poseen solamente efectos indirectos positivos sobre la variable dependiente $(\beta I T=.259 ; \beta I=.113$ y $\beta I=.291$ respectivamente $)$. Finalmente, las variables exógenas con un menor efecto sobre la variable dependiente fueron la edad $(\beta I T=.177)$, cuya relación con las actitudes hacia personas trans es positiva, y el nivel socioeconómico $(\beta I T=-.135)$ siendo la única variable independiente que posee una relación negativa con la variable endógena pura.

Tabla 2. Índices de adecuación del modelo re-especificado obtenido para la EANT con y sin ATGL

\begin{tabular}{lccc}
\hline ÍNDICE & $\begin{array}{c}\text { Valores de } \\
\text { ajuste } \\
\text { (con ATGL) }\end{array}$ & $\begin{array}{c}\text { Valores de } \\
\text { ajuste } \\
\text { (sin ATGL) }\end{array}$ & $\begin{array}{c}\text { Valores de } \\
\text { ajuste para } \\
\text { ANTGL }\end{array}$ \\
\hline $\boldsymbol{X}^{2}$ & 30.366 & 32.901 & 14.245 \\
$\mathbf{g l}$ & 14 & 11 & 14 \\
$\boldsymbol{X}^{2} / \mathrm{gl}$ & 2.169 & 2.991 & 1.018 \\
AGFI & .949 & .938 & .973 \\
CFI & .991 & .985 & .992 \\
PRATIO & .389 & .393 & .389 \\
RMSEA & .053 & .070 & .007 \\
AIC & 92.366 & 82.901 & 76.245 \\
\hline
\end{tabular}

Tabla de elaboración propia. 
Tabla 3. Efectos directos, indirectos y totales de variables independientes sobre la variable endógena pura EANTP

\begin{tabular}{|c|c|c|c|c|}
\hline & Efectos & Directos & Indirectos & Totales \\
\hline \multicolumn{5}{|l|}{ Variables exógenas } \\
\hline NSE & & .000 & -.135 & -.135 \\
\hline Edad & & .070 & .106 & .177 \\
\hline Intensidad religiosa & & .085 & .377 & .462 \\
\hline \multicolumn{5}{|l|}{$\begin{array}{l}\text { V. intermediarias: } \\
\text { conservadurismo }\end{array}$} \\
\hline$S D O D G$ & & .000 & .113 & .113 \\
\hline$R W A$ & & .000 & .291 & .291 \\
\hline \multicolumn{5}{|c|}{$\begin{array}{l}\text { V. intermediarias: } \\
\text { prejuicio sexual y de género }\end{array}$} \\
\hline Sexismo Hostil & & .000 & .259 & .259 \\
\hline Sexismo Benevolente & & .272 & .161 & .433 \\
\hline ATGL & & .515 & .000 & .515 \\
\hline
\end{tabular}

Tabla de elaboración propia. $\mathrm{N}=387$ (sólo heterosexuales)

\section{Conclusiones}

El presente trabajo permitió identificar las contribuciones y efectos directos e indirectos de variables sociodemográficas, intensidad del sentimiento religioso, conservadurismo político (RWA y SDO-Dominancia Grupal) y creencias y actitudes relacionadas con el orden de género y sexual (sexismo hostil y benevolente y ATGL), en las actitudes negativas hacia personas trans (EANT) en población heterosexual de la ciudad de Córdoba.

Los análisis aquí presentados sugieren que las actitudes negativas hacia personas trans se presentan como corolario de posiciones conservadoras en lo social, y se expresan en la concatenación de diversos prejuicios asociados a un orden sexual y de género tradicional.

Las personas más conservadores (quienes presentaron mayores puntajes en RWA y SDO en su dimensión de dominancia grupal), en la medida en que se presentan como más resistentes al cambio y a la variabilidad social, son más prejuiciosas hacia las personas trans. Como en otros contextos, el autoritarismo de ala derecha presenta significativo poder explicativo, dando la pauta de que los perfiles autoritarios tienden a expresar mayores niveles de prejuicio incluso cuando se indaga de manera manifiesta (Etchezahar, 2012; Nagoshi et al., 2008; Norton \& Herek, 2012, Rottenbacher, 2012). El menor impacto explicativo de la orientación a la dominancia social podría estar dando cuenta que los grupos de personas trans se perciben como relegados de la esfera social aunque podrían no concebirse como un grupo mayormente en competencia que afecte el status grupal cisgénero y/o heterosexual. No obstante, también hay que considerar la limitación de que SDO no presentó buenos indicadores de validez interna en su conjunto, y sólo pudo utilizarse el subfactor de orientación a la dominancia grupal.

La intensidad del sentimiento religioso no sólo presenta efectos directos en la EANTP, si no que contribuye también explicativamente a partir de sus efectos en RWA, SDO-Dominancia Grupal, el sexismo benevolente y las actitudes negativas hacia gays y lesbianas (Nagoshi et al., 2008; Norton \& Herek, 2012). En general, las variables que aluden a la religiosidad personal son las más referidas por los estudios de prejuicio sexual y hacia trans, debido a que los discursos religiosos ejercen influencia en cuestiones relativas a la sexualidad y, en la mayoría de las religiones universales, se defienden postulados fuertemente heteronormativos (Herek et al., 2007). Llamativamente, la intensidad religiosa no presentó efectos directos en el sexismo hostil, aunque sí en el benevolente. Esto permite pensar que las amenazas percibidas por parte de las personas más religiosas podría combinar a la vez una visión paternalista de las mujeres y creencias jerárquicas de valor sexual, siendo cada vez más las experiencias y demandas públicas de la diversidad sexual las que se conciben como mayormente disruptivas y competitivas al orden sexual y de género tradicional que buscan sostener.

En este sentido, tanto el sexismo como el prejuicio sexual se presentan como próximos al prejuicio hacia personas trans: las personas heterosexuales en las que se advierte mayores niveles de sexismo benevolente y un mayor rechazo hacia gays y lesbianas, también presentan actitudes negativas hacia trans.

Conceptualmente, una alta correlación entre ATGL y EANT, además de evidenciar potenciales problemas de multicolinealidad, podría implicar que las personas trans, de forma confusa, a menudo son percibidas como homosexuales, mientras que las personas homosexuales pueden se percibidas también como transgresoras de las normas binarias de género. De acuerdo a Norton y Herek (2012) las correlaciones significativas entre estas variables indicarían un fuerte vínculo psicológico entre los dos dominios de actitud, al que habría que sumar el sexismo benevolente, según reflejan los análisis. No obstante, la fuerza de la correlación no alcanzó los niveles identificados por algunos de nuestros antecedentes (Costa et al., 2016; Grigoropoulos \& Kourdoutis, 2015), a la par que fue similar a la reportada para el sexismo benevolente. Esta diferencia con estudios en Brasil y Grecia, podrían implicar un creciente reconocimiento social de las particularidades de cada segmento de la población LGBT, lo cual puede haberse visto impactado a su vez por los debates públicos en torno a los reconocimientos legales conquistados por los movimientos de diversidad sexual en el país en los últimos años.

Considerando que existen evidencias significativas que sostienen una concatenación de los prejuicios indagados, a la vez que ciertas diferencias en la intensidad de las asociaciones y efectos para cada caso, no es posible decantar por una perspectiva que suponga un prejuicio de género y sexual generalizado, como propusieron Kite y Whitley (1996) y desarrollaron en Brasil Costa et al. (2016), ni por una perspectiva que enfatice la diferencia- 
ción de cada tipo de prejuicio (Herek, Chopp \& Stroll, 2007; Worther, 2013). Un abordaje que enfatiza el peso de variables de religiosidad personal y el conservadurismo político, evidenciado en autoritarismo y anti-igualitarismo, no habilita per se identificar diferencias significativas en los factores explicativos de cada prejuicio indagado. Las actitudes negativas hacia las personas trans parecen tener sus raíces psicológicas en un alto apoyo a las convenciones sociales, las percepciones de jerarquías de poder y los valores tradicionales, expresados en la defensa de un orden sexual y de género tradicional. En este aspecto, son similares no sólo al prejuicio sexual sino a otras actitudes negativas hacia diversos exogrupos (Etchezahar, 2012; Rottenbacher et al., 2011). Por ejemplo, cuando la variable ATGL es descartada del modelo de path, RWA y religiosidad continúan siendo predictores significativos de las actitudes hacia personas trans. Probablemente la inclusión a futuro de variables como la necesidad de cierre cognitivo (Burke et al., 2017) o la distancia o contacto interpersonal con personas gays, lesbianas y trans (Herek \& Capitanio, 1996), permitan avanzar en mayores evidencias para sostener o rechazar alguna de las dos perspectivas. También se sugiere abordar con mayor especificidad las actitudes hacia derechos reclamados por colectivos trans, frente a aquellos más específicos de gays y lesbianas (Flores, 2015).

Consideramos que el presente estudio constituye un contribución necesaria al estudio de las actitudes sociales hacia persona trans, desde la tradición de investigación del prejuicio social, y con una muestra de población general. Aún se requiere avanzar en nuevas indagaciones con muestreos probabilísticos para identificar el impacto que los reconocimientos legales puedan tener en el reconocimiento social de las personas trans, una de las poblaciones más postergadas y vulneradas de Argentina.

\section{REFERENCIAS}

Altemeyer, B. (2004). Highly dominating, highly authoritarian personalities. The Journal of Social Psychology, 144(4), 421-448.

Asociación Argentina de Marketing, Sociedad Argentina de Investigación de Marketing y Opinión \& Cámara de Empresas de Investigación Social y de Mercado (2006, noviembre) NSE 2006. Recuperado de http://www.saimo.org.ar/archivos/observatorio-social/NSE2006-23nov2006-Informe-final.pdfo do preconceito contra diversidade sexual e de gênero: construção de um instrumento, Estudos de Psicologia, 32, 163-172.

Bazán, B. (diciembre, 2014). Subsidio, prejuicio e identidad de género. Revista Trama. Recuperado de http://tramarevista.com. ar/politica/subsidio-prejuicio-e-identidad-de-genero/

Burke, S. E., Dovidio, J. F., LaFrance, M., Przedworski, J. M., Perry, S. P., Phelan, S. M., ... van Ryn, M. (2017). Beyond generalized sexual prejudice: Need for closure predicts negative attitudes toward bisexual people relative to gay/lesbian people, Journal of Experimental Social Psychology, 71, 145-150.

Cárdenas, M. \& Barrientos, J. (2008). The Attitudes toward Lesbians and Gay Men Scale (ATLG): Adaptation and Testing the Reliability and Validity in Chile, Journal of Sex Research, 45(2), 140-149.
Cárdenas, M., Lay, S., González, C., Calderón, C., \& Alegría, I. (2010). Inventario de sexismo ambivalente: adaptación, validación, y relación con variables psicosociales. Revista Salud y Sociedad, 1(2), 125-135.

Costa, A. B., Machado, W., Bandeira, D. R. \& Nardi, H. C. (2016), Validation Study of the Revised Version of the Scale of Prejudice Against Sexual and Gender Diversity in Brazil, Journal of Homosexuality, 63(11), 1446-1463.

Costa, P. A., \& Davies, M. (2012). Portuguese adolescents' attitudes toward sexual minorities: transphobia, homophobia, and gender role beliefs. Journal of homosexuality, 59(10), 1424-1442.

Etchezahar, E. (2012). Las dimensiones del autoritarismo: análisis de la escala de autoritarismo del ala de derechas (RWA) en una muestra de estudiantes universitarios de la Ciudad de Buenos Aires. Psicologia Política, 12 (25), 591-603.

Etchezahar, E., Ungaretti, J., Prado, V. \& Brussino, S. (2016). Psychometric properties of the Attitudes Toward Gay men scale in Argentinian context: The influence of sex, authoritarianism, and social dominance orientation. International Journal of Psychological Research, 9, 21-29.

Flores, A. (2015). Attitudes toward transgender rights: perceived knowledge and secondary interpersonal contact. Politics, groups and identities. 3(3), 398-416.

Glick, P., \& Fiske, S. T. (1996). The Ambivalent Sexism Inventory: Differentiating hostile and benevolent sexism. Journal of personality and social psychology, 70(3), 491.

Grasmick, H. G., Cochran, J. K., Bursik Jr, R. J., \& Kimpel, M. L. (1993). Religion, punitive justice, and support for the death penalty. Justice Quarterly, 10(2), 289-314.

Grigoropoulos, I. \& Kordoutis, P. (2015). Social Factors Affecting Antitransgender Sentiment in a Sample of Greek Undergraduate Students, International Journal of Sexual Health, 27(3), 276-285.

Herek, G. (1988). Heterosexuals' Attitudes toward Lesbians and Gay Men: Correlates and Gender Differences. The Journal of Sex Research, 25, 451-477.

Herek, G. (1994). Beyond Homophobia: A social psychological perspective in attitudes toward lesbians and gay men. Journal of Homosexuality, 10, 2-17.

Herek, G. (2000). The psychology of sexual prejudice. Current Directions in Psychological Science, 9(1), 19-22.

Herek, G. (2002). Gender Gaps in Public Opinion about Lesbians and Gay Men. The Public Opinion Quarterly, 66(1), 40-66.

Herek, G. M. \& Capitanio, J. P. (1996). "Some of my best friends": Intergroup contact, concealable stigma, and heterosexuals' attitudes toward gay men and lesbians. Personality and Social Psychology Bulletin, 22, 412-424.

Herek, G., Chopp, R., \& Strohl, D. (2007). Sexual stigma: Putting sexual minority health issues in context. En I. Meyer \& M. Northridge (Eds.), The health of sexual minorities: Public health perspectives on LGBT populations (pp, 171-208). New York, NY: Springer.

Hill, D. \& Willoughby, B. (2005). The development and validation of the genderism and transphobia scale. Sex Roles, 53, 531-544.

Hill, D. B. (2002). Genderism, transphobia, and gender bashing: A framework for interpreting anti-transgender violence. En: B. Wallace \& R. Carter (Eds.), Understanding and dealing with violence: A multicultural approach (pp.113-136). Thousand Oaks, CA: Sage. 
Hill, E. D., Cohen, A. B., Terrell, H. K., \& Nagoshi, C. T. (2010). The role of social cognition in the religious fundamentalismprejudice relationship. Journal for the Scientific Study of Religion, 49(4), 724-739.

Imhoff, D. \& Brussino, S. (2013). Análisis psicométrico de la dimensión autoritarismo general de la escala RWA en Córdoba/ Argentina. Revista Avances en Medición, 8, 67-79.

Instituto Nacional de Estadísticas y Censos [INDEC]. (2012). Primera Encuesta Sobre Población Trans 2012: Travestis, Transexuales, Transgéneros y Hombres Trans. Informe Técnico de la Prueba Piloto Municipio de la Matanza. Recuperado de http:// www.trabajo.gov.ar/downloads/diversidadsexual/Argentina_Primera_Encuesta_sobre_Poblacion_Trans_2012.pdf

Iosa, T., Rabbia, H., Sgró Ruata, M.C, Morán Faúndes, J. M. \& Vaggione, J.M. (2012). Política, sexualidades y derechos. Primera Encuesta Marcha del Orgullo y la Diversidad Córdoba, Argentina 2010. Rio de Janeiro, BR: CEPESC. Recuperado de http:// www.clam.org.br/uploads/arquivo/Pol\%C3\%ADtica,\%20sexualidades $\% 20$ y $\% 20$ derechos $\% 20$ - \% 20 Marcha $\% 20$ C\%C3\%B3rdoba.pdf

Jaume, L. C., Etchezahar, E. D., Cervone, N. C., \& Biglieri, J. (diciembre, 2011). Adaptación y validación de la escala Orientación a la dominancia Social (SDO) al contexto argentino mediante AFC. Trabajo presentado en el V Congreso Marplatense de Psicología, Universidad Nacional de Mar del Plata, Argentina.

Johnson, M. K., Rowatt, W. C., Barnard-Brak, L. M., Patock-Peckham, J. A., LaBouff, J. P., \& Carlisle, R. D. (2011). A mediational analysis of the role of right-wing authoritarianism and religious fundamentalism in the religiosity-prejudice link. Personality and Individual Differences, 50(6), 851-856.

Jorge, J. E. (2012). Same-sex marriage in Argentina tolerance and discrimination in political culture. Journal of Research in Peace, Gender and Development, 2(3), 60-71.

Jost, J. T., Glaser, J., Kruglanski, A. W., \& Sulloway, F. J. (2003). Political conservatism as motivated social cognition. Psychological bulletin, 129(3), 339.

Kite, M. \& Whitley, B.E. (1996). Sex Differences in Attitudes Toward Homosexual Persons, Behaviors, and Civil Rights A Meta-Analysis. Personality and Social Psychology Bulletin, 22(4), 336-353.

Kline, R. B. (2011). Principles and practice of structural equation modeling. New York, NY: The Guilford Press.

Landén, M., \& Innala, S. (2000). Attitudes toward transsexualism in a Swedish national survey. Archives of Sexual Behavior, 29(4), 375-388.

Lista, C. (1995). ¿Existe consenso hacia el aborto inducido?: tendencias de la población de la ciudad de Córdoba. Córdoba: Universidad Nacional de Córdoba.

Lohr, S. (2000). Muestreo: diseño y análisis. México: Thompson.

Moral de la Rubia, J. (2010). Religión, significados y actitudes hacia la sexualidad: Un enfoque psicosocial, Rev. Colombiana de Psicología, 19(1), 45-59.

Nagoshi, J., Adams, K., Terrell, H., Hill, E., Brzuzy, S. \& Nagoshi, C. (2008). Gender Differences in Correlates of Homophobia and Transphobia. Sex Roles, 59, 521-531.
Norton, A. T., \& Herek, G. M. (2012). Heterosexuals' attitudes toward transgender people: Findings from a national probability sample of US adults. Sex roles, 68(11-12), 738-753.

Páez, J., Hevia, G., Pesci, F. \& Rabbia, H.H. (2015). Construcción y validación de una escala de actitudes negativas hacia personas trans. Revista de Psicología (PUCP), 33(1), 152-188.

Pearl, M. L., \& Galupo, M. P. (2007). Development and validation of the attitudes toward same-sex marriage scale. Journal of homosexuality, 53(3), 117-134.

Pérez, E., Medrano, L. A., \& Sánchez Rosas, J. (2013). El path analysis: conceptos básicos y ejemplos de aplicación. Revista Argentina de Ciencias del Comportamiento, 5(1), 52-66.

Pérez-Tejeda, H. E. (2007). Estadística, ciencias sociales, del comportamiento y de la salud. Ciudad de México, MX: Cengage Learning Editores.

Petit, L., \& Costa, G. L. (2011). Dominancia social: El género como jerarquía social. Psicológica, 9(1), 161-168.

Poteat, V. P., \& Mereish, E. H. (2012). Ideology, prejudice, and attitudes toward sexual minority social policies and organizations. Political Psychology, 33(2), 211-224.

Pratto, F., Sidanius, J., Stallworth, L., \& Malle, B. F. (1994). Social dominance orientation: A personality variable predicting social and political attitudes Journal of Personality and Social Psychology, 67(4), 741-763.

Rodríguez Otero, L. M. \& Treviño Martínez, L. (2016). Sexismo y actitudes hacia la homosexualidad, bisexualidad y la transexualidad en estudiantes de trabajo social mexicanos. Trabajo Social Global, 6(11), 3-33.

Rottenbacher J. M. (2012). Conservadurismo político, homofobia y prejuicio hacia grupos transgénero en una muestra de estudiantes y egresados universitarios de Lima. Pensamiento Psicológico, 10(1), 23-37.

Rottenbacher, J. M., Espinosa, A. \& Magallanes, J. M. (2011). Analizando el prejuicio: Bases ideológicas del racismo, el sexismo y la homofobia en una muestra de habitantes de la ciudad de Lima-Perú. Revista Psicologia Política, 11(22), 225-246.

Schmall, E. (25 de mayo de 2012). Transgender Advocates Hail Law Easing Rules in Argentina, The New York Times. Recuperado de https://www.nytimes.com/2012/05/25/world/americas/transgender-advocates-hail-argentina-law.html

Seoane, J. \& Garzón, A. (1992). Creencias Sociales Contemporáneas, autoritarismo y humanismo. Psicología Política, 5, 27-52.

Theumer, E. (septiembre, 2018). Del género como ideología. La Tinta. Recuperado de https://latinta.com.ar/2018/09/generocomo-ideologia/

Whitley, B.E. \& Ægisdottir, S. (2000). The Gender Belief System, Authoritarianism, Social Dominance Orientation, and Heterosexuals' Attitudes Toward Lesbians and Gay Men. Sex Roles, 42(11/12), 947-967.

Worthen, M. (2013). An Argument for Separate Analyses of Attitudes Toward Lesbian, Gay, Bisexual Men, Bisexual Women, MtF and FtM Transgender Individuals. Sex Roles, 68(11-12), 703-72. 